\title{
Advances in depression research: second special issue, 2020, with highlights on biological mechanisms, clinical features, co-morbidity, genetics, imaging, and treatment
}

\author{
Julio Licinio ${ }^{1} \cdot$ Ma-Li Wong ${ }^{1}$
}

(c) Springer Nature Limited 2020

The current speed of progress in depression research is simply remarkable. We have therefore been able to create a second special issue of Molecular Psychiatry, 2020, focused on depression, with highlights on mechanisms, genetics, clinical features, co-morbidity, imaging, and treatment. We are also very proud to present in this issue a seminal paper by Chottekalapanda et al., which represents some of the last work conducted by the late Nobel Laureate Paul Greengard [1]. This brings to four the number of papers co-authored by Paul Greengard and published in our two 2020 depression special issues [1-4].

The research content of this special depression issue starts with Chottekalapanda et al.'s outstanding contribution aimed at determining whether neuroadaptive processes induced by antidepressants are modulated by the regulation of specific gene expression programs [1]. That team identified a transcriptional program regulated by activator protein-1 (AP-1) complex, formed by c-Fos and c-Jun that is selectively activated prior to the onset of the chronic SSRI response. The AP-1 transcriptional program modulated the expression of key neuronal remodeling genes, including S100a10 (p11), linking neuronal plasticity to the antidepressant response. Moreover, they found that AP-1 function is required for the antidepressant effect in vivo. Furthermore, they demonstrated how neurochemical pathways of BDNF and FGF2, through the MAPK, PI3K, and JNK cascades, regulate AP-1 function to mediate the beneficial effects of the antidepressant response. This newly identified molecular network provides "a new avenue that

Julio Licinio

juliolicinio@gmail.com

1 State University of New York, Upstate Medical University, Syracuse, NY 13210, USA could be used to accelerate or potentiate antidepressant responses by triggering neuroplasticity."

A superb paper by Schouten et al. showed that oscillations of glucocorticoid hormones (GC) preserve a population of adult hippocampal neural stem cells in the aging brain [5]. Moreover, major depressive disorder (MDD) is characterized by alterations in GC-related rhythms [6, 7]. GC regulate neural stem/precursor cells (NSPC) proliferation $[8,9]$. The adrenals secrete GC in ultradian pulses that result in a circadian rhythm. GC oscillations control cell cycle progression and induce specific genome-wide DNA methylation profiles. Schouten et al. studied primary hippocampal NSPC cultures and showed that GC oscillations induced lasting changes in the methylation state of a group of gene promoters associated with cell cycle regulation and the canonical Wnt signaling pathway. Furthermore, in a mouse model of accelerated aging, they showed that disruption of GC oscillations induced lasting changes in dendritic complexity, spine numbers and morphology of newborn granule neurons. Their results indicate that GC oscillations preserve a population of GR-expressing NSPC during aging, preventing their activation possibly by epigenetic programming through methylation of specific gene promoters. These important observations suggest a novel mechanism mediated by GC that controls NSPC proliferation and preserves a dormant NSPC pool, possibly contributing to neuroplasticity reserve in the aging brain.

MDD has a critical interface with addiction and suicide, which is of immense clinical and research importance [10]. Peciña et al. have reviewed a growing body of research indicating that the endogenous opioid system is directly involved in the regulation of mood and is dysregulated in MDD [11]. Halikere et al. provide evidence that addiction associated N40D mu-opioid receptor variant modulates synaptic function in human neurons [12].

Two papers by Amare et al. and Coleman et al. examine different genetic substrates for MDD, identifying novel 
depression-related loci as well as studying the interface with trauma [13, 14].

The dissection of MDD clinical phenotypes, including their interface with other illnesses is a topic of several articles in this special issue. Belvederi Murri et al. examined the symptom network structure of depressive symptoms in late-life in a large European population in the 19 country Survey of Health, Ageing, and Retirement in Europe (SHARE) (mean age 74 years, 59\% females, $n=8557$ ) [15]. They showed that the highest values of centrality were in the symptoms of death wishes, depressed mood, loss of interest, and pessimism. Another article focused on a specific feature of MDD, namely changes in appetite. Simmons et al. aimed at explaining why some individuals lose their appetite when they become depressed, while others eat more, and brought together data on neuroimaging, salivary cortisol, and blood markers of inflammation and metabolism [16]. Depressed participants experiencing decreased appetite had higher cortisol levels than other subjects, and their cortisol values correlated inversely with the ventral striatal response to food cues. In contrast, depressed participants experiencing increased appetite exhibited marked immunometabolic dysregulation, with higher insulin, insulin resistance, leptin, c-reactive protein (CRP), interleukin 1 receptor antagonist (IL-1RA), and IL-6, and lower ghrelin than subjects in other groups, and the magnitude of their insulin resistance correlated positively with the insula response to food cues. Their findings support the existence of pathophysiologically distinct depression subtypes for which the direction of appetite change may be an easily measured behavioral marker.

Mulugeta et al. studied the association between major depressive disorder and multiple disease outcomes in the UK Biobank $(n=337,536)$ [17]. They performed hypothesis-free phenome-wide association analyses between MDD genetic risk score (GRS) and 925 disease outcomes. MDD was associated with several inflammatory and hemorrhagic gastrointestinal diseases, and intestinal $E$. coli infections. MDD was also associated with disorders of lipid metabolism and ischemic heart disease. Their results indicated a causal link between MDD and a broad range of diseases, suggesting a notable burden of co-morbidity. The authors concluded that "early detection and management of MDD is important, and treatment strategies should be selected to also minimize the risk of related co-morbidities." Further information on the shared mechanisms between coronary heart disease and depression in the UK Biobank $(n=367,703)$ was explored by Khandaker et al. [18]. They showed that family history of heart disease was associated with a $20 \%$ increase in depression risk; however, a genetic risk score that is strongly associated with CHD risk was not associated with depression. Their data indicate that comorbidity between depression and CHD arises largely from shared environmental factors.
In a systematic review and meta-analysis of cohort studies, Wang et al. examined the interface of depression and anxiety in relation to cancer incidence and mortality [19]. Their analyses suggest that depression and anxiety may have an etiologic role and prognostic impact on cancer, although there is potential reverse causality.

Several papers in this issue examine imaging in MDD, either to unravel the underlying disease processes or to identify imaging biomarkers of treatment response. Let us first look at the studies focused on elucidating brain circuitry alterations in MDD. Arterial spin labeling (ASL) was used by Cooper et al. to measure cerebral blood flow (CBF; perfusion) in order to discover and replicate alterations in CBF in MDD [20]. Their analyses revealed reduced relative $\mathrm{CBF}(\mathrm{rCBF})$ in the right parahippocampus, thalamus, fusiform, and middle temporal gyri, as well as the left and right insula, for those with MDD. They also revealed increased $\mathrm{rCBF}$ in MDD in both the left and the right inferior parietal lobule, including the supramarginal and angular gyri. According to the authors, "these results (1) provide reliable evidence for ASL in detecting differences in perfusion for multiple brain regions thought to be important in MDD, and (2) highlight the potential role of using perfusion as a biosignature of MDD." Further data on imaging in MDD was provided by a coordinated analysis across 20 international cohorts in the ENIGMA MDD working group. In that paper, van Velzen et al. showed that in a coordinated and harmonized multisite diffusion tensor imaging study there were subtle, but widespread differences in white matter microstructure in adult MDD, which may suggest structural disconnectivity [21].

Four articles in this special issue examine imaging biomarkers of treatment response. Greenberg et al. studied reward-related ventral striatal activity and differential response to sertraline versus placebo in depressed using functional magnetic resonance imaging while performing a reward task [22]. They found that ventral striatum (VS) dynamic response to reward expectancy (expected outcome value) and prediction error (difference between expected and actual outcome), likely reflecting serotonergic and dopaminergic deficits, was associated with better response to sertraline than placebo. Their conclusion was that treatment measures of reward-related VS activity may serve as objective neural markers to advance efforts to personalize interventions by guiding individual-level choice of antidepressant treatment. Utilizing whole-brain functional connectivity analysis to identify neural signatures of remission following antidepressant treatment, and to identify connectomic predictors of treatment response, Korgaonkar et al. showed that intrinsic connectomes are a predictive biomarker of remission in major depressive disorder [23]. Based on their results that team proposed that increased functional connectivity within and between large-scale intrinsic brain networks may characterize 
acute recovery with antidepressants in depression. Repple et al. created connectome matrices via a combination of T1-weighted magnetic resonance imaging (MRI) and tractography methods based on diffusion-weighted imaging severity of current depression and remission status in 464 MDD patients and 432 healthy controls [24]. Reduced global fractional anisotropy (FA) was observed specifically in acute depressed patients compared to fully remitted patients and healthy controls. Within the MDD patients, FA in a subnetwork including frontal, temporal, insular, and parietal nodes was negatively associated with symptom intensity, an effect remaining when correcting for lifetime disease severity. Their findings provide new evidence of MDD to be associated with structural, yet dynamic, state-dependent connectome alterations, which covary with current disease severity and remission status after a depressive episode. The effects of electroconvulsive therapy (ECT), the most effective treatment for depression, on the dentate gyrus (DG) were studied by Nuninga et al. through an optimized MRI scan at 7-tesla field strength, allowing sensitive investigation of hippocampal subfields [25, 26]. They documented a large and significant increase in DG volume after ECT, while other hippocampal subfields were unaffected. Furthermore, an increase in DG volume was related to a decrease in depression scores, and baseline DG volume predicted clinical response. These findings suggest that the volume change of the DG is related to the antidepressant properties of ECT, possibly reflecting neurogenesis.

Three articles report new directions for antidepressant therapeutics. Papakostas et al. presented the results of a promising phase 2 , double-blind, placebo-controlled study of NSI-189 phosphate, a novel neurogenic compound, in MDD patients [27]. As the endogenous opioid system is thought to play an important role in the regulation of mood, Fava et al. studied the buprenorphine/samidorphan combination as an investigational opioid system modulator for adjunctive treatment of MDD in two phase 3, randomized, double-blind, placebo-controlled studies that utilized the same sequential parallel-comparison design [28]. One of the studies achieved the primary endpoint, namely change from baseline in Montgomery-Åsberg Depression Rating Scale (MADRS)-10 at week 5 versus placebo) and the other study did not achieve the primary endpoint. However, the pooled analysis of the two studies demonstrated consistently greater reduction in the MADRS-10 scores from baseline versus placebo at multiple timepoints, including end of treatment. These data provide cautious optimism and support further controlled trials for this potential new treatment option for patients with MDD who have an inadequate response to currently available antidepressants. Fava et al. also report the results of a double-blind, placebo-controlled, dose-ranging trial of intravenous (IV) ketamine as adjunctive therapy in treatment-resistant depression, using four doses of ketamine and a control [29, 30]. They show that there was evidence for the efficacy of the two higher doses of IV ketamine and no clear or consistent evidence for clinically meaningful efficacy of the two lower doses studied.

Overall, in this issue, immense progress in depression research is provided by outstanding studies that highlight advances in our understanding of MDD biology, clinical features, co-morbidity, genetics, brain imaging (including imaging biomarkers), and treatment. Building on the groundbreaking articles from our previous 2020 special issues on stress and behavior [31-49] and on depression [2$4,50-62]$, we are proud that the stunning progress presented here found its home in our pages. From inception in 1996, we have aimed at making Molecular Psychiatry promote the integration of molecular medicine and clinical psychiatry [63]. It is particularly rewarding to see that goal achieved so spectacularly in this second 2020 special issue on MDD, a disorder of gene-environment interactions that represents a pressing public health challenge, with an ever increasing impact on society [64-66]. We are privileged to have in these two 2020 depression special issues four remarkable papers from Paul Greengard's teams that provide substantial new data on the mechanisms of antidepressant action [1-4]. Such profound advances in basic science are needed to facilitate and guide future translational efforts needed to advance therapeutics [67, 68].

\section{References}

1. Chottekalapanda R, et al. AP-1 controls the p11-dependent antidepressant response. Mol Psychiatry. 2020. https://doi.org/ 10.1038/s41380-020-0767-8.

2. Sagi Y, et al. Emergence of 5-HT5A signaling in parvalbumin neurons mediates delayed antidepressant action. Mol Psychiatry. 2019. https://doi.org/10.1038/s41380-019-0379-3.

3. Oh SJ, et al. Hippocampal mossy cell involvement in behavioral and neurogenic responses to chronic antidepressant treatment. Mol Psychiatry. 2019. https://doi.org/10.1038/s41380-019-0384-6.

4. Shuto T, et al. Obligatory roles of dopamine D1 receptors in the dentate gyrus in antidepressant actions of a selective serotonin reuptake inhibitor, fluoxetine. Mol Psychiatry. 2018. https://doi. org/10.1038/s41380-018-0316-x.

5. Schouten M, et al. Circadian glucocorticoid oscillations preserve a population of adult hippocampal neural stem cells in the aging brain. Mol Psychiatry. 2019. https://doi.org/10.1038/s41380-019-0440-2.

6. Kling MA, et al. Effects of electroconvulsive therapy on the CRHACTH-cortisol system in melancholic depression: preliminary findings. Psychopharmacol Bull. 1994;30:489-94.

7. Sternberg EM, Licinio J. Overview of neuroimmune stress interactions. Implications for susceptibility to inflammatory disease. Ann NY Acad Sci. 1995;771:364-71.

8. Bornstein SR, et al. Stress-inducible-stem cells: a new view on endocrine, metabolic and mental disease? Mol Psychiatry. 2019;24:2-9. https://doi.org/10.1038/s41380-018-0244-9.

9. Rubin de Celis MF, et al. The effects of stress on brain and adrenal stem cells. Mol Psychiatry. 2016;21:590-3. https://doi.org/10. 1038/mp.2015.230. 
10. Soares-Cunha $\mathrm{C}$, et al. Nucleus accumbens medium spiny neurons subtypes signal both reward and aversion. Mol Psychiatry. 2019. https://doi.org/10.1038/s41380-019-0484-3.

11. Pecina $M$, et al. Endogenous opioid system dysregulation in depression: implications for new therapeutic approaches. Mol Psychiatry. 2019;24:576-87, https://doi.org/10.1038/s41380018-0117-2.

12. Halikere A, et al. Addiction associated N40D mu-opioid receptor variant modulates synaptic function in human neurons. Mol Psychiatry. 2019. https://doi.org/10.1038/s41380019-0507-0.

13. Amare AT, et al. Bivariate genome-wide association analyses of the broad depression phenotype combined with major depressive disorder, bipolar disorder or schizophrenia reveal eight novel genetic loci for depression. Mol Psychiatry. 2019. https://doi.org/ 10.1038/s41380-018-0336-6.

14. Coleman JRI, et al. Genome-wide gene-environment analyses of major depressive disorder and reported lifetime traumatic experiences in UK Biobank. Mol Psychiatry. 2020 https://doi.org/10. 1038/s41380-019-0546-6.

15. Belvederi Murri M, Amore M, Respino M, Alexopoulos GS. The symptom network structure of depressive symptoms in late-life: results from a European population study. Mol Psychiatry. 2018. https://doi.org/10.1038/s41380-018-0232-0.

16. Simmons WK, et al. Appetite changes reveal depression subgroups with distinct endocrine, metabolic, and immune states. Mol Psychiatry. 2018. https://doi.org/10.1038/s41380-018-0093-6.

17. Mulugeta A, Zhou A, King C, Hypponen E. Association between major depressive disorder and multiple disease outcomes: a phenome-wide Mendelian randomisation study in the UK Biobank. Mol Psychiatry. 2019. https://doi.org/10.1038/s41380-019-0486-1.

18. Khandaker GM, et al. Shared mechanisms between coronary heart disease and depression: findings from a large UK general population-based cohort. Mol Psychiatry. 2019. https://doi.org/10. 1038/s41380-019-0395-3.

19. Wang $\mathrm{YH}$, et al. Depression and anxiety in relation to cancer incidence and mortality: a systematic review and meta-analysis of cohort studies. Mol Psychiatry. 2019. https://doi.org/10.1038/ s41380-019-0595-x.

20. Cooper CM, et al. Discovery and replication of cerebral blood flow differences in major depressive disorder. Mol Psychiatry. 2019. https://doi.org/10.1038/s41380-019-0464-7.

21. van Velzen LS, et al. White matter disturbances in major depressive disorder: a coordinated analysis across 20 international cohorts in the ENIGMA MDD working group. Mol Psychiatry. 2019. https://doi.org/10.1038/s41380-019-0477-2.

22. Greenberg T, et al. Reward related ventral striatal activity and differential response to sertraline versus placebo in depressed individuals. Mol Psychiatry. 2019. https://doi.org/10.1038/s41380-0190490-5.

23. Korgaonkar MS, Goldstein-Piekarski AN, Fornito A \& Williams, LM Intrinsic connectomes are a predictive biomarker of remission in major depressive disorder. Mol Psychiatry. 2019. https://doi. org/10.1038/s41380-019-0574-2.

24. Repple J, et al. Severity of current depression and remission status are associated with structural connectome alterations in major depressive disorder. Mol Psychiatry. 2019. https://doi.org/10. 1038/s41380-019-0603-1.

25. Nuninga JO, et al. Volume increase in the dentate gyrus after electroconvulsive therapy in depressed patients as measured with 7T. Mol Psychiatry. 2019. https://doi.org/10.1038/s41380-019-0392-6.

26. Koch SBJ, Morey RA \& Roelofs K. The role of the dentate gyrus in stress-related disorders. Mol Psychiatry. 2019. https://doi.org/ 10.1038/s41380-019-0572-4.

27. Papakostas GI, et al. A phase 2, double-blind, placebo-controlled study of NSI-189 phosphate, a neurogenic compound, among outpatients with major depressive disorder. Mol Psychiatry. 2019. https://doi.org/10.1038/s41380-018-0334-8.

28. Fava M, et al. Opioid system modulation with buprenorphine/ samidorphan combination for major depressive disorder: two randomized controlled studies. Mol Psychiatry. 2018. https://doi. org/10.1038/s41380-018-0284-1.

29. Fava M, et al. Correction: double-blind, placebo-controlled, doseranging trial of intravenous ketamine as adjunctive therapy in treatment-resistant depression (TRD). Mol Psychiatry. 2019. https://doi.org/10.1038/s41380-018-0311-2.

30. Fava M, et al. Double-blind, placebo-controlled, dose-ranging trial of intravenous ketamine as adjunctive therapy in treatmentresistant depression (TRD). Mol Psychiatry. 2018. https://doi.org/ 10.1038/s41380-018-0256-5 (2018).

31. Licinio J. Advances in research on stress and behavior: special issue, 2020. Mol Psychiatry 2020;25:916-7. https://doi.org/10. 1038/s41380-020-0741-5.

32. Martinez ME, et al. Thyroid hormone overexposure decreases DNA methylation in germ cells of newborn male mice. Mol Psychiatry. 2020;25:915 https://doi.org/10.1038/s41380-020-0732-6.

33. Martinez ME, et al. Thyroid hormone influences brain gene expression programs and behaviors in later generations by altering germ line epigenetic information. Mol Psychiatry. 2020;25:939-50. https://doi.org/10.1038/s41380-018-0281-4.

34. Le-Niculescu H, et al. Towards precision medicine for stress disorders: diagnostic biomarkers and targeted drugs. Mol Psychiatry. 2020;25:918-38. https://doi.org/10.1038/s41380-019-0370-z.

35. Torres-Berrio A, et al. MiR-218: a molecular switch and potential biomarker of susceptibility to stress. Mol Psychiatry. 2020;25:951-64. https://doi.org/10.1038/s41380-019-0421-5

36. Sillivan SE, et al. Correction: MicroRNA regulation of persistent stress-enhanced memory. Mol Psychiatry. 2020;25:1154 https:// doi.org/10.1038/s41380-019-0452-y.

37. Sillivan SE, et al. MicroRNA regulation of persistent stressenhanced memory. Mol Psychiatry. 2020;25:965-76. https://doi. org/10.1038/s41380-019-0432-2.

38. Shi MM, et al. Hippocampal micro-opioid receptors on GABAergic neurons mediate stress-induced impairment of memory retrieval. Mol Psychiatry. 2020;25:977-92. https://doi.org/10.1038/s41380019-0435-z.

39. Mayo LM, et al. Protective effects of elevated anandamide on stress and fear-related behaviors: translational evidence from humans and mice. Mol Psychiatry. 2020;25:993-1005. https://doi. org/10.1038/s41380-018-0215-1.

40. Qu N, et al. A POMC-originated circuit regulates stress-induced hypophagia, depression, and anhedonia. Mol Psychiatry. 2020;25:1006-21. https://doi.org/10.1038/s41380-019-0506-1.

41. Fox ME, et al. Dendritic remodeling of D1 neurons by RhoA/ Rho-kinase mediates depression-like behavior. Mol Psychiatry. 2020;25:1022-34. https://doi.org/10.1038/s41380-018-0211-5.

42. Jin J, et al. Ahnak scaffolds p11/Anxa2 complex and L-type voltage-gated calcium channel and modulates depressive behavior. Mol Psychiatry. 2020;25:1035-49. https://doi.org/10.1038/ s41380-019-0371-y.

43. Ben-Yehuda $\mathrm{H}$, et al. Maternal Type-I interferon signaling adversely affects the microglia and the behavior of the offspring accompanied by increased sensitivity to stress. Mol Psychiatry. 2020;25:1050-67. https://doi.org/10.1038/s41380-019-0604-0.

44. Pearson-Leary J, et al. The gut microbiome regulates the increases in depressive-type behaviors and in inflammatory processes in the ventral hippocampus of stress vulnerable rats. Mol Psychiatry. 2020;25:1068-79. https://doi.org/10.1038/s41380-019-0380-x.

45. Walker WH 2nd, et al. Acute exposure to low-level light at night is sufficient to induce neurological changes and depressive-like behavior. Mol Psychiatry. 2020;25:1080-93. https://doi.org/10. 1038/s41380-019-0430-4. 
46. Lei Y, et al. SIRT1 in forebrain excitatory neurons produces sexually dimorphic effects on depression-related behaviors and modulates neuronal excitability and synaptic transmission in the medial prefrontal cortex. Mol Psychiatry. 2020;25:1094-111. https://doi.org/10.1038/s41380-019-0352-1.

47. Sargin D, et al. Mapping the physiological and molecular markers of stress and SSRI antidepressant treatment in S100a10 corticostriatal neurons. Mol Psychiatry. 2020;25:1112-29. https://doi.org/ 10.1038/s41380-019-0473-6.

48. Iob E, Kirschbaum C, Steptoe A. Persistent depressive symptoms, HPA-axis hyperactivity, and inflammation: the role of cognitiveaffective and somatic symptoms. Mol Psychiatry. 2020;25:1130-40. https://doi.org/10.1038/s41380-019-0501-6.

49. Cabeza de Baca T, et al. Chronic psychosocial and financial burden accelerates 5-year telomere shortening: findings from the Coronary Artery Risk Development in Young Adults Study. Mol Psychiatry. 2020;25:1141-53. https://doi.org/10.1038/s41380-019-0482-5.

50. Licinio J \& Wong ML. Advances in depression research: special issue, 2020, with three research articles by Paul Greengard. Mol Psychiatry. 2020;25:1156-58. https://doi.org/10.1038/s41380020-0781-x.

51. Teissier A, et al. Early-life stress impairs postnatal oligodendrogenesis and adult emotional behaviour through activitydependent mechanisms. Mol Psychiatry. 2019. https://doi.org/10. 1038/s41380-019-0493-2.

52. Zhang Y, et al. CircDYM ameliorates depressive-like behavior by targeting miR-9 to regulate microglial activation via HSP90 ubiquitination. Mol Psychiatry. 2018. https://doi.org/10.1038/s41380018-0285-0.

53. Tan A, et al. Effects of the KCNQ channel opener ezogabine on functional connectivity of the ventral striatum and clinical symptoms in patients with major depressive disorder. Mol Psychiatry. 2018. https://doi.org/10.1038/s41380-018-0283-2.

54. Kin K, et al. Cell encapsulation enhances antidepressant effect of the mesenchymal stem cells and counteracts depressive-like behavior of treatment-resistant depressed rats. Mol Psychiatry. 2018. https://doi. org/10.1038/s41380-018-0208-0.

55. Orrico-Sanchez A, et al. Antidepressant efficacy of a selective organic cation transporter blocker in a mouse model of depression. Mol Psychiatry. 2019. https://doi.org/10.1038/s41380-019-0548-4.

56. Han Y, et al. Systemic immunization with altered myelin basic protein peptide produces sustained antidepressant-like effects. Mol Psychiatry. 2019. https://doi.org/10.1038/s41380-019-0470-9.
57. Wittenberg GM, et al. Effects of immunomodulatory drugs on depressive symptoms: a mega-analysis of randomized, placebocontrolled clinical trials in inflammatory disorders. Mol Psychiatry. 2019. https://doi.org/10.1038/s41380-019-0471-8.

58. Beydoun MA, et al. Systemic inflammation is associated with depressive symptoms differentially by sex and race: a longitudinal study of urban adults. Mol Psychiatry. 2019. https://doi.org/10. 1038/s41380-019-0408-2.

59. Felger JC, et al. What does plasma CRP tell us about peripheral and central inflammation in depression? Mol Psychiatry. 2018. https://doi.org/10.1038/s41380-018-0096-3.

60. Clark SL, et al. A methylation study of long-term depression risk. Mol Psychiatry. 2019. https://doi.org/10.1038/s41380-0190516-z.

61. Aberg KA, et al. Methylome-wide association findings for major depressive disorder overlap in blood and brain and replicate in independent brain samples. Mol Psychiatry. 2018. https://doi.org/ 10.1038/s41380-018-0247-6.

62. Wei $\mathrm{YB}$, et al. A functional variant in the serotonin receptor 7 gene (HTR7), rs7905446, is associated with good response to SSRIs in bipolar and unipolar depression. Mol Psychiatry. 2019. https://doi.org/10.1038/s41380-019-0397-1.

63. Licinio J. Molecular Psychiatry: the integration of molecular medicine and clinical psychiatry. Mol Psychiatry. 1996;1:1-3.

64. Steenblock C, et al. Severe acute respiratory syndrome coronavirus 2 (SARS-CoV-2) and the neuroendocrine stress axis. Mol Psychiatry. 2020. https://doi.org/10.1038/s41380020-0758-9.

65. Wong ML, Dong C, Andreev V, Arcos-Burgos M, Licinio J. Prediction of susceptibility to major depression by a model of interactions of multiple functional genetic variants and environmental factors. Mol Psychiatry. 2012;17:624-33. https://doi.org/ 10.1038/mp.2012.13.

66. Lee SH, Paz-Filho G, Mastronardi C, Licinio J, Wong ML. Is increased antidepressant exposure a contributory factor to the obesity pandemic? Transl Psychiatry. 2016;6:e759 https://doi.org/ 10.1038/tp.2016.25.

67. Bornstein SR, Licinio J. Improving the efficacy of translational medicine by optimally integrating health care, academia and industry. Nat Med. 2011;17:1567-9. https://doi.org/10.1038/ nm. 2583 .

68. Licinio J, Wong ML. Launching the 'war on mental illness'. Mol Psychiatry. 2014;19:1-5. https://doi.org/10.1038/mp.2013.180. 